\title{
Disinhibition of the Nucleus Accumbens Leads to Macro-Scale Hyperactivity Consisting of Micro-Scale Behavioral Segments Encoded by Striatal Activity
}

\author{
Dorin Yael, @Orel Tahary, Boris Gurovich, Katya Belelovsky, and ๑Izhar Bar-Gad \\ The Leslie and Susan Goldschmied (Gonda) Multidisciplinary Brain Research Center, Bar-Ilan University, Ramat-Gan, 52900, Israel
}

\begin{abstract}
The striatum comprises of multiple functional territories involved with multilevel control of behavior. Disinhibition of different functional territories leads to territory-specific hyperkinetic and hyperbehavioral symptoms. The ventromedial striatum, including the nucleus accumbens (NAc) core, is typically associated with limbic input but was historically linked to high-level motor control. In this study, performed in female Long-Evans rats, we show that the NAc core directly controls motor behavior on multiple timescales. On the macro-scale, following NAc disinhibition, the animals manifested prolonged hyperactivity, expressed as excessive normal behavior, whereas on the micro-scale multiple behavior transitions occurred, generating short movement segments. The underlying striatal network displayed population-based local field potential transient deflections (LFP spikes) whose rate determined the magnitude of the hyperactivity and whose timing corresponded to unitary behavioral transition events. Individual striatal neurons preserved normal baseline activity and network interactions following the disinhibition, maintaining the normal encoding of behavioral primitives and forming a sparse link between the LFP spikes and single neuron activity. Disinhibition of this classically limbic territory leads to profound motor changes resembling hyperactivity and attention deficit. These behavioral and neuronal results highlight the direct interplay on multiple timescales between different striatal territories during normal and pathological conditions.
\end{abstract}

Key words: attention deficit hyperactivity disorder; GABA; ADHD; locomotion; nucleus accumbens; ventral striatum

Significance Statement

The nucleus accumbens (NAc) is a key part of the striatal limbic territory. In the current study we show that this classically limbic area directly controls motor behavior on multiple timescales. Focal disinhibition of the NAc core in freely behaving rats led to macro-scale hyperactivity and micro-scale behavioral transitions, symptoms typically associated with attention deficit hyperactivity disorder. The behavioral changes were encoded by the striatal LFP signal and single-unit spiking activity in line with the neuronal changes observed during tic expression following disinhibition of the striatal motor territory. These results point to the need to extend the existing parallel functional pathway concept of basal ganglia function to include the study of limbic-motor cross-territory interactions in both health and disease.

\section{Introduction}

The striatum, the main input structure of the corticobasal ganglia pathway, receives converging glutamatergic inputs organized in multiple mostly-parallel pathways: sensorimotor afferents terminate primarily in the dorsolateral striatum, whereas limbic affer-

\footnotetext{
Received Dec. 13, 2018; revised May 14, 2019; accepted May 16, 2019.

Author contributions: D.Y. and I.B.-G. designed research; D.Y. and I.B.-G. performed research; D.Y., 0.T., B.G., K.B., and I.B.-G. contributed unpublished reagents/analytic tools; D.Y. and I.B.-G. analyzed data; D.Y. and I.B.-G. wrote the paper.

This study was supported in part by an Israel Science Foundation Grant (297/18) and a BSF-NSF Collaborative Research in Computational Neuroscience Grant (2016744).

The authors declare no competing financial interests.

Correspondence should be addressed to Izhar Bar-Gad at izhar.bar-gad@biu.ac.il.

https://doi.org/10.1523/JNEUROSCI.3120-18.2019

Copyright $\odot 2019$ the authors
}

ents target the ventromedial striatum and specifically the nucleus accumbens (NAc; McGeorge and Faull, 1989; Heilbronner et al., 2016). The NAc is further subdivided based on the anatomical division to the core and shell (Voorn et al., 1989; Heimer et al., 1991). Although the separation into territories is widely accepted, the specific functional role of individual territories and subterritories is under debate. Recent studies of the NAc have focused on limbic functions such as reward, decision making, and addiction (Al-Hasani et al., 2015; Volkow and Morales, 2015; Floresco et al., 2018). Specifically, the core is associated primarily in goal directed behavior, whereas the shell is associated with motivational values (West and Carelli, 2016). However, earlier work emphasized the NAc role in bridging between the limbic and motor systems (Mogenson et al., 1980) and more specifically, 
control over overall activity in general, and locomotion in particular (Mogenson and Nielsen, 1984). This control over locomotion may be experimentally modulated by focal pharmacological manipulation of glutamate (Arnt, 1981; Swanson and Kalivas, 2000), dopamine (Costall and Naylor, 1975; Jones et al., 1981; Costall et al., 1984), and the extensive GABAergic network of the NAc (Morgenstern et al., 1984; Yoshida et al., 1991). Moreover, this modulation is affected by the interactions between the different pharmacological agents (Pycock and Horton, 1979; Dalia et al., 1998) leading to various hyperactive and hypoactive states. The NAc control over movement is primarily modulated by the NAc core as demonstrated by both lesion and pharmacological manipulations (Maldonado-Irizarry and Kelley, 1994, 1995).

These functional differences between striatal territories are apparent in the expression of a myriad of hyperkinetic and hyperbehavioral conditions involving the different territories. Tourette syndrome, a childhood onset neurological disorder characterized by the expression of motor and vocal tics, is primarily associated with abnormalities in the sensorimotor regions of the corticobasal ganglia pathway (Sowell et al., 2008; Tremblay et al., 2015). Attention deficit hyperactivity disorder (ADHD), a neurodevelopmental disorder characterized by attention difficulties, hyperactivity, and impulsivity, primarily involves limbic parts of the circuit (Giedd et al., 2006; Qiu et al., 2009). These two disorders show high comorbidity rates, in that $>50 \%$ of all Tourette syndrome patients express comorbid ADHD (Schlaggar and Mink, 2003), hinting that the two disorders may share some common underlying pathophysiology. Disinhibition of different functional territories of the striatum by focal blocking of $\mathrm{GABA}_{\mathrm{A}}$ transmission in rodents and primates supports this hypothesis. Disinhibition leads to the expression of territory specific hyperkinetic and hyper-behavioral symptoms; namely, motor tics following disinhibition of the motor territory (Tarsy et al., 1978; McCairn et al., 2009; Bronfeld et al., 2013) and hyperactivity following similar manipulation in the limbic territories (Morgenstern et al., 1984; Yoshida et al., 1991; Worbe et al., 2009).

In this study we use disinhibition to deconstruct the behavioral elements controlled by the NAc core on multiple timescales and identify the underlying neurophysiological mechanism encoding these elements. The relation between behavior and striatal activity has been explored during in the dorsolateral striatum during normal behavior (Klaus et al., 2017; Markowitz et al., 2018) and disinhibition (Bronfeld et al., 2011; Israelashvili and Bar-Gad, 2015; Oran and Bar-Gad, 2018) but is lacking for the ventral striatum during either states. This neuronal-behavioral relationship may serve as a basis for establishing the unique role of the NAc in the control of movement in normal and hyperkinetic conditions.

\section{Materials and Methods}

\section{Animals}

Fifteen adult female Long-Evans rats (weighing $288.5 \pm 28.4 \mathrm{~g}$, mean \pm SD) were used in this study. The animals were kept in a temperature and humidity controlled room with a $12 \mathrm{~h}$ light/dark cycle and had ad libitum access to food and water. Experiments were performed during the light phase in a lighted setup. All procedures were approved and supervised by the Institutional Animal Care and Use Committee and were in accordance with the National Institute of Health Guide for the Care and Use of Laboratory Animals and the Bar-Ilan University Guidelines for the Use and Care of Laboratory Animals in Research. This study was approved by the National Committee for Experiments in Laboratory Animals at the Israel Ministry of Health.

\section{Surgical procedures}

The animals were implanted unilaterally with an injection cannula targeting the NAc core (AP: 1.5, ML: 2.2, DV: 7.0) and a recording electrode array (custom-made movable bundles of 16/32 Formvar isolated nichrome microwires, $25 \mu \mathrm{m}$ diameter, A-M Systems) in the ventromedial striatum (AP: 0.5, ML: 2.2, DV: 5.0-7.0). In four animals, additional recording electrodes were placed in the dorsolateral striatum (AP: 0.5 , ML: 4.0, DV: 3.5-5.5). All the surgical procedures were described in detail in previous studies by our group (Bronfeld et al., 2013; Yael et al., 2013; Israelashvili and Bar-Gad, 2015; Oran and Bar-Gad, 2018).

\section{Experimental sessions}

Following a recovery period lasting at least $7 \mathrm{~d}$ post-operation, the animals were connected to a wireless recording system and were placed in one of three behavioral arenas allowing free natural behavior: (1) open field $(80 \times 120 \mathrm{~cm}),(2)$ limited field $(80 \times 120 \mathrm{~cm}$, containing six $20 \times$ $20 \mathrm{~cm}$ cubes), and (3) linear field $(8 \times 180 \mathrm{~cm})$. The animals' neuronal activity was continuously recorded and their behavior was monitored using a 50/60 fps rate video camera and 9-axis kinematic sensors located within the wireless acquisition system. Following a $30 \mathrm{~min}$ recording period during the naive state, the animals were removed from the behavioral arena and bicuculline methiodide was injected unilaterally into their NAc core. After the injection, the animals were returned to the arena, and their neuronal activity and behavior were recorded for an additional $30-45 \mathrm{~min}$. The behavioral changes induced by the microinjection followed the temporal dynamics of the bicuculline spread and clearance from the tissue, in line with previous studies of disinhibition using bicuculline in the sensorimotor striatum (Bronfeld et al., 2013). At the end of the experiment and after return to normal behavior, the animals were returned to their home cage.

\section{Microinjections}

Unilateral micro-injections of the GABA-A antagonist bicuculline methiodide into the NAc core were performed with an acute injector (30 G stainless steel tube attached to a flexible Tygon microbore tube), which was manually inserted into a chronically implanted guiding cannula ( 25 $\mathrm{G}$ stainless steel tube) located $2 \mathrm{~mm}$ above the injection target. Bicuculline methiodide (Sigma-Aldrich) was dissolved in artificial CSF [concentrations (in mM): $145 \mathrm{NaCl}, 15 \mathrm{HEPES}, 2.5 \mathrm{KCl}, 2 \mathrm{MgCl}_{2}, 1.2 \mathrm{CaCl}_{2}, \mathrm{PH}$ 7.4 with $\mathrm{NaOH}$ ] at a concentration of $1 \mu \mathrm{g} / \mu \mathrm{l}$. A volume of $0.5 \mu \mathrm{l}$ was injected at a rate of $0.5 \mu \mathrm{g} / \mathrm{min}$ using a $10 \mu \mathrm{l}$ syringe (Hamilton) attached to a programmable syringe pump (NE-1000, New Era Pump Systems). One minute after the injection termination, the injector was removed and the guiding cannula was sealed by a cannula-dummy ( $28 \mathrm{G}$ stainless steel wire). The injection procedure, volume and concentration were similar to those previously used during bicuculline injections to the sensorimotor striatum (Bronfeld et al., 2013).

\section{Neuronal recordings and preprocessing}

Electrophysiological signals were continuously recorded during the session using either a 16- or 32-channel wireless acquisition system (NeuroLog-16 and RatLog-64, Deuteron Technologies). The analog signal was bandpass filtered $(1 \mathrm{~Hz}$ one-pole to 7000 or $10,000 \mathrm{~Hz}$ three-pole Butterworth filter), amplified $(\times 200)$, and digitized (12 or 16 bits with a resolution of 3.3 or $0.2 \mu \mathrm{V}$, sampling rates: 29.297 or $32 \mathrm{kHz}$, respectively). Off-line, the recording files were concatenated, filtered (300$6000 \mathrm{~Hz}, 4$-pole bidirectional Butterworth filter), and sorted for the extraction of single-unit spike times (Offline sorter, Plexon). This approach, in which the recording files are concatenated to create a single long file including the full experimental phase, allowed sorting the whole period using the same criteria and examination of the unit stability throughout the session. The suspected spikes were cut out of the streams in $2 \mathrm{~ms}$ windows and consequently projected over multiple parameters (such as the PCs and maximal and minimal values). Only units which formed a clear cluster, displayed a clean refractory period $(<0.1 \%$ of the spikes occurring within short intervals) and which maintained the same waveform were included in the final dataset. The LFP signal was extracted using off-line low-pass filtration of the raw digitized signal (cutoff frequency: $300 \mathrm{~Hz}$, 4-pole bidirectional Butterworth filter). To avoid filterbased distortion of waveforms (Yael and Bar-Gad, 2017) and timing 
information (Yael et al., 2018), all the off-line filters used in the study were bidirectional (zero-phase) filters. All subsequent analyses were performed using custom-written code (MATLAB, MathWorks).

\section{Data analysis}

Rat location detection. Video recordings were off-line frame-by-frame analyzed to extract the 2-dimensional location of the animals. Detection used custom written MATLAB code based on a background subtraction algorithm. After subtraction of the background frame, individual frames were converted from RGB into grayscale coding. Using Otsu's method (Otsu, 1979), frames were further reduced from grayscale to a binary BW image. The binary image was then smoothed by eroding with a 2-dimensional disk shaped structuring object. Noise was subtracted by the removal of connected components $<600$ pixels. Finally, centroids were extracted and confirmed to be within a radius of 200 pixels from the centroid detected in the previous frame. The software and documentation are available via GIT (https://github.com/neurintlab/ratDetect).

Distance and velocity calculation. After the extraction of the 2dimensional rat location, the coordinates over time were convolved with a Gaussian window ( $S D=2$ frames). The Euclidean distance between successive coordinates was calculated, converted from pixels to metric units, and normalized by the frame rate to evaluate the corresponding velocity. The velocity was then smoothed using Gaussian window.

Auto and cross-correlations. Spike train-based auto and crosscorrelations were calculated for pairs of simultaneously recorded neurons, for a stable period of $10 \mathrm{~min}$ starting $10 \mathrm{~min}$ from the beginning of the session (pre-injection and postinjection states). The crosscorrelation functions were only calculated for neurons recorded on different channels to avoid artificial correlations (Bar-Gad et al., 2001). The cross-correlation functions were calculated within a time window of $\pm 4.5 \mathrm{~s}$ with a time resolution of $1 \mathrm{~ms}$ and were smoothed using a Gaussian window ( $\mathrm{SD}=40 \mathrm{~ms}) . Z$-scores were calculated based on a baseline period located $\pm 2-4 \mathrm{~s}$ from zero time lag. The correlation was considered significant if the integral of a time window of $\pm 10 \mathrm{~ms}$ normalized by the number of bins crossed the confidence levels $(p<0.01)$. Based on this significance test, the cross-correlation functions were then classified into one of three groups: positive, negative, or no correlation.

Raster plots, PLTHs, and joint PLTHs. To evaluate the spiking activity around local field potential transient deflections (LFP spikes), peri-LFPspike rasters were calculated by aligning $2000 \mathrm{~ms}$ windows extracted from the single-unit spike trains to the LFP spike onset. To calculate the peri-LFP-spike time histograms (PLTHs), the rasters were averaged and smoothed by a Gaussian window (SD $25 \mathrm{~ms}$ ).

To estimate the temporal relationship between two spike trains around the LFP spike times we calculated the joint PLTHs (JPLTHs). Pairs of spike trains (bin size: $1 \mathrm{~ms}$ ) based on simultaneously recorded neurons were used to create a matrix in which all the correlated firing combinations within \pm 1000 ms window for each event were summed. This matrix was corrected by subtraction of the shift-predicator matrix (calculated by multiplication of the corresponding PLTHs; Aertsen et al., 1989) and smoothed using 2D convolution with a Gaussian window $(\mathrm{SD}=50 \mathrm{~ms})$. To evaluate the time dependent coactivation of the singleunit pairs, we extracted the main diagonal of the corrected matrix corresponding to the coactivation of the neurons around the LFP within the window with zero time lags. Sparse matrices were manually removed from downstream calculations to avoid possible artifacts. The significance of the response was estimated by calculating the confidence lines $(p<0.001)$ based on baseline activity $( \pm 400-900 \mathrm{~ms}$ from the LFP spike onset). JPLTHs were considered to have a significant response if they crossed the significance lines in a time window of $\pm 50 \mathrm{~ms}$ around the LFP spikes onset.

Movement termination detection. Movement terminations were detected using a threshold-crossing algorithm combined with trough detection based on the animals' velocity (smoothed by a $100 \mathrm{~ms}$ SD Gaussian window). The threshold was set to the mean velocity $-0.2 \times$ $\mathrm{SD}$ of the velocity. Events below threshold and local minima $>1$ SD of the velocity were identified when the inter-event intervals were limited to $200 \mathrm{~ms}$. The detected movement termination train was used to calculate peri-LFP spike-triggered stop histograms.
Event triggered velocity. The spike-triggered velocity was calculated for a period of $15 \mathrm{~min}$ starting $10 \mathrm{~min}$ after the initiation of pre- and postinjection states. The single unit/LFP spike train and its corresponding velocity (smoothed by a $100 \mathrm{~ms}$ SD Gaussian window) were synchronized. The event-triggered velocity within a time window of $\pm 5 \mathrm{~s}$ was calculated by averaging the $z$-scores of the velocity around individual events. Confidence levels were calculated by a baseline period located in the first 1000 $\mathrm{ms}$ of the window. The relationship to behavior for significant eventtriggered velocities was determined by the deviation of the velocity around the event onset (positive deviation, movement initiation; peak, peak velocity; negative, movement termination).

Statistical analysis. The statistical hypotheses were assessed using either normal distribution tests or nonparametric tests in cases of non-normal distributions (such as intervals) depending on the specific dataset. The values are presented as either mean \pm SD or mean \pm SEM as stated in the text. The resulting $\mathrm{p}$ values are shown in raw format. In all cases, the tests were performed using their standard MATLAB implementation without any modifications.

\section{Histology}

At completion of experimental sessions, the animals were deeply anesthetized using a mixture of ketamine $(100 \mathrm{mg} / \mathrm{kg})$, xylazine $(10 \mathrm{mg} / \mathrm{kg})$, and morphinhydrochlorid $(0.15 \mathrm{mg} / \mathrm{kg})$. The microinjection location within the NAc core was marked using an injection of $0.5 \mu \mathrm{l}$ of $1 \%$ toluidine blue and was used as the inclusion criterion. The rats were transcardially perfused with $0.9 \%$ saline and $10 \%$ paraformaldehyde and their brains were removed and stored in a fixation solution of $30 \%$ sucrose and $10 \%$ paraformaldehyde. The implanted structures were preserved and examined to confirm their relative location using $50 \mu \mathrm{m}$ coronal sections.

\section{Results}

\section{Behavioral effect: hyperactivity}

The $\mathrm{GABA}_{\mathrm{A}}$ antagonist bicuculline was unilaterally injected into the NAc core of freely behaving rats (Fig. 1A). We conducted a total of 34 recording sessions in 15 rats, which included a naive (pre-injection) period followed by the microinjection, and the subsequent postinjection period. The movement of the rats was assessed with a combination of automatic detection using a single video stream and 3D motion sensors (accelerometers and gyroscopes). The injection led to a hyperactive state, which was expressed as increased normal behavior without abnormal motor symptoms (Movie 1). The overall ambulation of the rat increased significantly following the injection (Fig. $1 B$ ). The overall distance traveled increased significantly $(n=23$, pre: $54.9 \pm 5.9 \mathrm{~m}$, post: $156.8 \pm 13.7 \mathrm{~m}$, mean $\pm \mathrm{SEM}$ over a period of $25 \mathrm{~min}, p=$ $3.6 \times 10^{-9}$, paired $t$ test). This increase was highly dependent on the activity of the animal before the injection, such that highly active naive animals tended to increase their activity after the injection to a larger extent than less active ones (Pearson's correlation coefficient: $0.65, p=0.0007$; Fig. $1 C$ ). The bicuculline injection led to a prolonged exploration period in which the animals continuously ambulated in the arena. This contrasted sharply with the naive state, in which following a short period of exploration the level of ambulation decreased (Fig. 1D-F). Following the injection, the animals spent a higher fraction of time in active periods (Fig. $1 G ; n=23$, Pre: $0.48 \pm 0.05$, Post: $0.90 \pm$ 0.02 , mean \pm SEM over a period of $25 \mathrm{~min}, p=8.4 \times 10^{-3}$, paired $t$ test) and moved faster during these active periods (Fig. $1 H ; n=23$, Pre: $3.65 \pm 0.15 \mathrm{~cm} / \mathrm{s}$, Post: $6.41 \pm 0.46 \mathrm{~cm} / \mathrm{s}$, mean \pm SEM over only the active periods, $p=6.6 \times 10^{-7}$, paired $t$ test) compared with the pre-injection state. The animals' velocity postinjection was positively correlated with their velocity before the injection (Pearson's correlation coefficient on logarithmic values: $0.59, p=0.0032$; Fig. $1 H$ ). This hyperactivity was expressed bilaterally, thus, the animals did not display a prefer- 
A

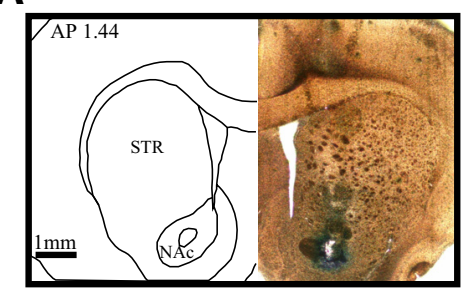

C

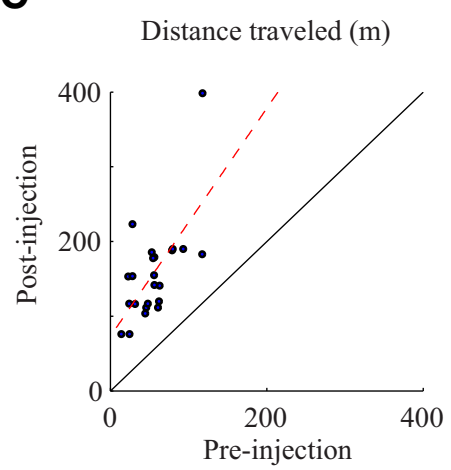

F

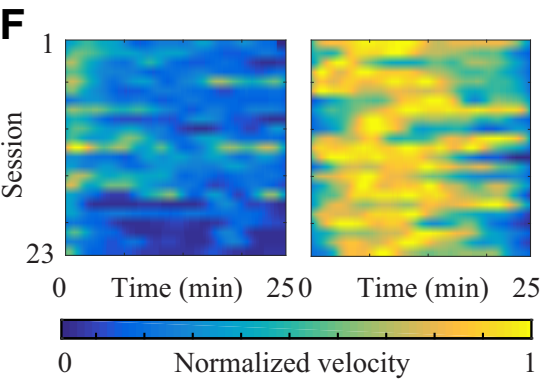

D

E
B

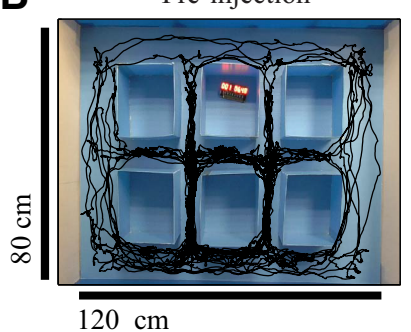

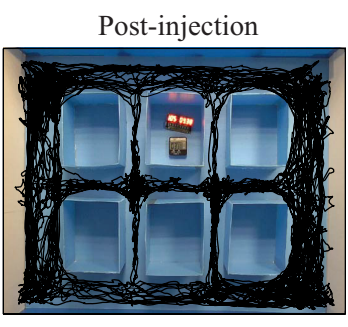
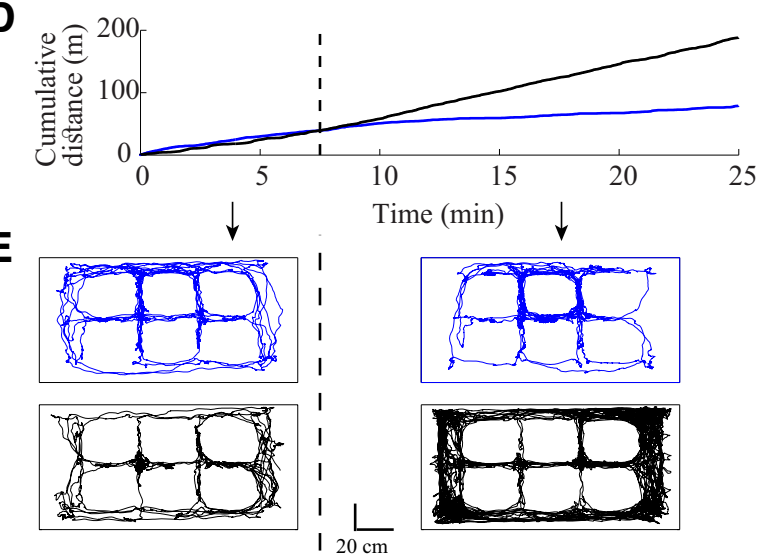

G

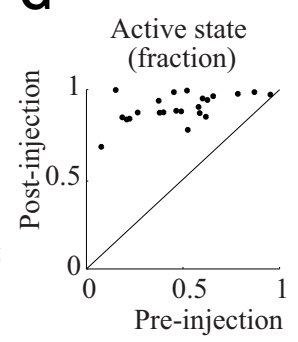

H

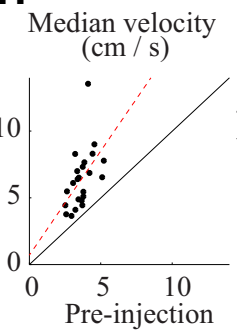

I

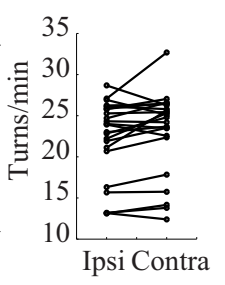

Figure 1. Behavioral effects of NAc core disinhibition. $A$, The location of bicuculline injection within the NAc marked on a coronal section of the rat brain (right) and the corresponding atlas-based section (AP: 1.44; left). $\boldsymbol{B}$, Rat tracks $>25$ min pre- (left) and post- (right) injection of a single session. C, The overall distance traveled over 25 min pre- and post-injection during all sessions (dashed red line, linear regression). $\boldsymbol{D}$, Cumulative distance traveled pre- (blue) and post- (black) injection during a single session (dashed black line, point of separation). $\boldsymbol{E}$, The rat track pre- (blue) and post(black) injection separated to before (left) and following the (right) point of separation. $\boldsymbol{F}$, Velocity over time during all the sessions, pre- (left) and post- (right) bicuculline injection normalized to the maximal velocity within each session. $\boldsymbol{G}, \boldsymbol{H}$, Relation between pre- and post-injection periods: $(\boldsymbol{G})$ fraction of active state of the whole session and $(\boldsymbol{H})$ median velocity. The solid black lines in $\boldsymbol{C}$, $\boldsymbol{G}$, and $\boldsymbol{H}$ mark the identity relationship. $\boldsymbol{I}$, Number of ipsiversive and contraversive turns postinjection calculated for a period of $25 \mathrm{~min}$ for all sessions.

ence for the ipsilateral or contralateral sides of the injection hemisphere (turns/min: ipsilateral: $22.27 \pm 0.99$; contralateral: $23.10 \pm 1.04$, mean \pm SEM, paired $t$ test: $p=0.56$; Fig. $1 I)$. In a few sessions, the animals developed short tetanic seizures or unilateral symptoms such as circling ipsilateral to the injection side, and motor tics contralateral to the injection hemisphere, potentially due to diffusion of bicuculline along the cannula (McCairn et al., 2009). Sessions in which the animals expressed motor tics $(n=4)$ and sessions with no apparent behavioral effect (probably because of malfunction of the injection process, $n=7$ ), were not included in the dataset.

\section{Neuronal correlates: hyperactivity}

The wideband filtered extracellular electrophysiological signal was continuously recorded from the striatum throughout the session. For the analysis of single-unit activity, the wideband signal was off-line high-pass filtered and single-unit spike times were extracted. Based on the action potential waveform, firing rate and pattern, the single units were classified as either spiny projection neurons [SPNs; also termed medium spiny neurons;
Pre: $n=106$, Post: $n=91$ ], tonically active neurons (TANs; Pre: $n=31$, Post: $n=19$ ), or fast-spiking interneurons (FSIs; Pre: $n=$ 13, Post: $n=17)$. The overall activity patterns of individual neurons and their interactions did not change following the injection despite the dramatic increase in the animals' behavior. The firing rates (Pre: SPN: $0.85 \pm 0.09$ TAN: $5.06 \pm 0.52$ FSI: $25.38 \pm 3.03$, Post: SPN: $1.15 \pm 0.13$ TAN: $6.07 \pm 0.89$ FSI: $23.17 \pm 2.56$ spikes/s, mean \pm SEM; $t$ test: SPN: $p=0.06$, TAN: $p=0.3$, FSI: $p=0.6$; Fig. $2 A$ ), firing patterns, as assessed by the coefficient of variation $(\mathrm{CV}$; Pre: SPN: $2.45 \pm 0.09$ TAN: $2.17 \pm 0.13$ FSI: $1.69 \pm 0.23$, Post: SPN: $2.55 \pm 0.10$ TAN: $2.10 \pm 0.19$ FSI: $1.55 \pm$ 0.13 , mean \pm SEM, $t$ test: SPN: $p=0.43$, TAN: $p=0.78$, FSI: $p=$ 0.58 ; Fig. $2 B$ ) and autocorrelation functions (Fig. $2 C$ ) were unaltered following the bicuculline injection. In line with the preservation of single-unit activity properties, the inter-striatal correlations were not significantly modulated by the bicuculline injection (SPN-SPN: Pre: $n=315$ pairs, Positive correlation: 93, negative correlation: 6; Post: $n=396$ pairs, Positive correlation: 127, negative correlation: 4; SPN-TAN: Pre: $n=231$ pairs, Positive correlation: 56 , negative correlation: 13 , Post: $n=175$ pairs, 


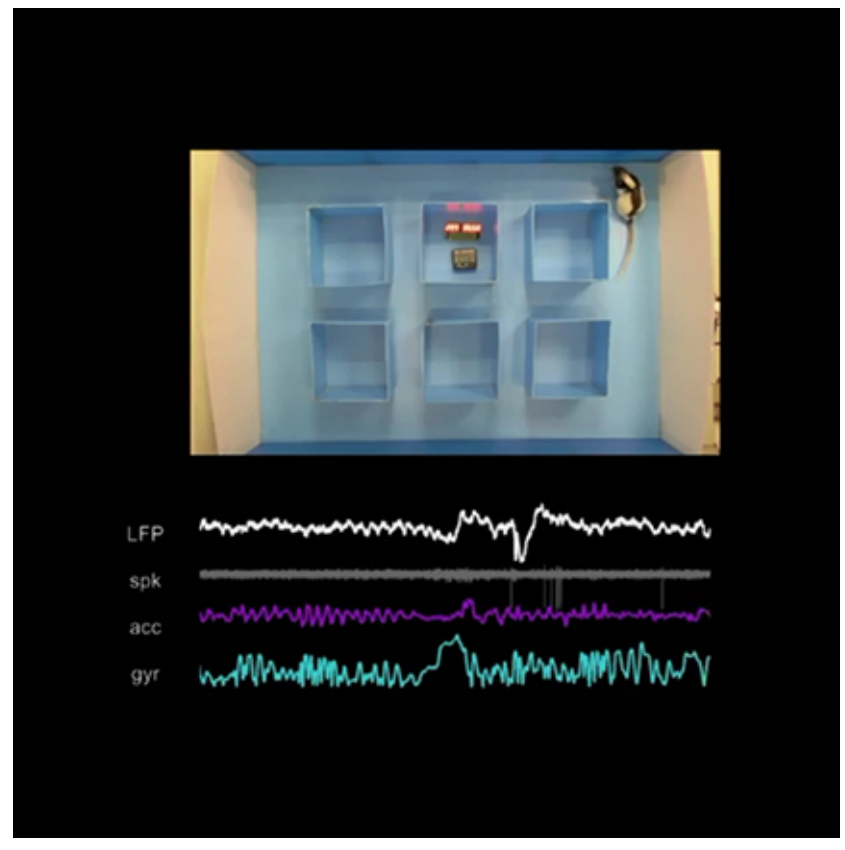

Movie 1. Effect of NAc bicuculline injection on behavior and neuronal activity. Behavior of the rat during the pre- (naive) and post- (hyperactive) injection states in the same session. A synchronized example of a single-channel LFP and spiking activity and the corresponding accelerometer and gyroscope signals, which are overlaid on the video stream.

Positive correlation: 29, negative correlation: 14; SPN-FSI: Pre: $n=84$, Positive correlation: 18 , negative correlation: 8 , Post: $n=$ 175 pairs, Positive correlation: 32 , negative correlation: $14 ; \chi^{2}$ values: SPN-SPN: 1.43, SPN-TAN: 4.02, SPN-FSI: 0.62, for all groups: $p>0.05$; Fig. $2 D$ ).

In a sharp contrast to the preserved properties of individual neurons, the injection led to changes in the LFP, which were expressed as large deflections (Fig. $3 A$ ). These deflections, termed LFP spikes, were characterized by a stereotypic shape which gradually developed over a few minutes following the injection, stabilized for a prolonged period, and finally decayed (Fig. $3 B$ ). The shape of the LFP spike was highly stereotypic during the stable period (Fig. 3C). During the stable periods, the LFP spike occurrences typically followed a Poisson distribution, which was reflected as an exponential distribution of the inter LFP spike intervals, with a refractory period (Fig. 3D). The rate of the LFP spikes varied across animals with a mean rate of $0.44 \mathrm{~Hz}$ (Fig. 3E). The rate of LFP spikes was highly reflective of the animals' state: the LFP spike rate and the behavioral effect strength (ratio of the distance traveled postinjection to the distance traveled preinjection) were highly correlated (Pearson's correlation coefficient, $r=0.83, p=0.001$; Fig. $3 F)$.

The activity of individual neurons was phase-locked to the LFP spikes across all the subpopulations (Fig. 4A, B). Although all the recorded neurons displayed a modulation in their activity, the temporal structure of the modulation was diverse, including multiple phases of excitation and inhibition with a variety of time constants (Fig. 4B). In most cases, the neurons displayed a prolonged inhibition, which was preceded, in some cases, by a short early excitation. This typical response was also evident in the mean response across the different neuronal populations (Fig. $4 B)$. Despite the common coactivation with the LFP spikes, the neuronal activity was not correlated in its responses. We calcu- lated the JPLTHs of simultaneously recorded pairs of single units ( $n=230$ pairs with non-sparse matrices; Fig. $4 C$ ). Only a small fraction displayed correlated activity around the time of the LFP spike (15/230, 6.5\%; Fig. 4D) indicating sparse locked neuronal activation around individual LFP spikes. These results demonstrate the translation of LFP information into a sparse finelytimed representation in the single-unit activity which is sufficient to drive the significant behavioral changes without changing in the striatal firing rates.

\section{Microscale behavior and neuronal activity}

On the macro-scale, NAc core disinhibition caused an increase in normal behavior prolonging the active state. A closer examination of the dynamics of behavior revealed a complex temporal microstructure consisting of bouts of rapid movements separated by brief cessation of locomotion, which was apparent in both the video-based and the sensor-based movement analysis (Fig. 5A). The neuronal activity was correlated to this micro-structure in that individual LFP spikes appeared at the time of locomotion termination (Movie 2; Fig. 5B). The peri-LFP spike movement stop histograms (Fig. $5 C$ ) and the LFP spike-triggered velocity (Fig. 5D) reflected this correlation between the behavioral changes and the LFP signal. The correlation between the LFP spikes and movement stops was only partial, whereas not all of the LFP spikes were accompanied by movement termination (Fig. 5B). To test whether the LFP spikes that were related or unrelated to movement termination were similar, we compared the temporal structure of the two populations (Fig. 5E), and calculated their mean shape $(F)$ and interval distribution $(G, H)$. None of these measures was significantly different for the two conditions (Interval distribution, Wilcoxon signed rank test, $p=$ 0.05 ; Mean shape: $t$ test performed on the first principal component, $p>0.05$ for all cases), suggesting that they represented events emerging from similar neuronal populations.

The behavioral micro-structure was accompanied by changes in the activity of individual neurons. Whereas the LFP spikes were highly associated with movement termination, individual neuron activity was correlated with additional components of movement, including movement initiation and the peak velocity (Fig. $6 A, B)$ covering multiple parts of each of the behavioral bouts. Correlations of this type have only been reported in neurons from the sensorimotor territories directly encoding motor behavior (Schultz and Romo, 1988; Cui et al., 2013) and were expanded to describe discrete actions or behavioral syllables (Klaus et al., 2017; Markowitz et al., 2018). This relationship to different behavioral components was found in all the striatal subpopulations (SPN: $n=66$ related: $36,54 \%$; TAN: $n=23$, related: $13,56 \%$; FSI: $n=31$, related: $18,58 \%$; fraction of movement related neurons: SPN, initiation: $14 \%$ peak velocity: $56 \%$, termination: $22 \%$, other: $8 \%$; TAN: initiation: $15 \%$, peak velocity: $46 \%$, termination: $31 \%$, other: $8 \%$; FSI: initiation: $6 \%$, peak velocity: $61 \%$, termination: $22 \%$, other: $11 \%$; Fig. $6 \mathrm{C}$ ). The fraction of neurons related to different parts of behavior did not differ pre- and postbicuculline injection (Pre: $n=58$ neurons, movement related: 35, 59\% movement unrelated: 23, 41; Post: $n=64$, movement related: $34,53 \%$, movement unrelated: $30,47 \% ; \chi^{2}$ test $=0.65$, $p>0.05$; Fig. $6 D$ ). Thus, despite the major change in behavior following the injection, the encoding of the behavioral microstructure by individual neurons did not change.

Single-unit activity was correlated with both LFP spikes and behavior. We explored this three-way interaction by partitioning the LFP spikes based on their relationship to movement termination and calculating the peri-LFP spike histograms for each 
A

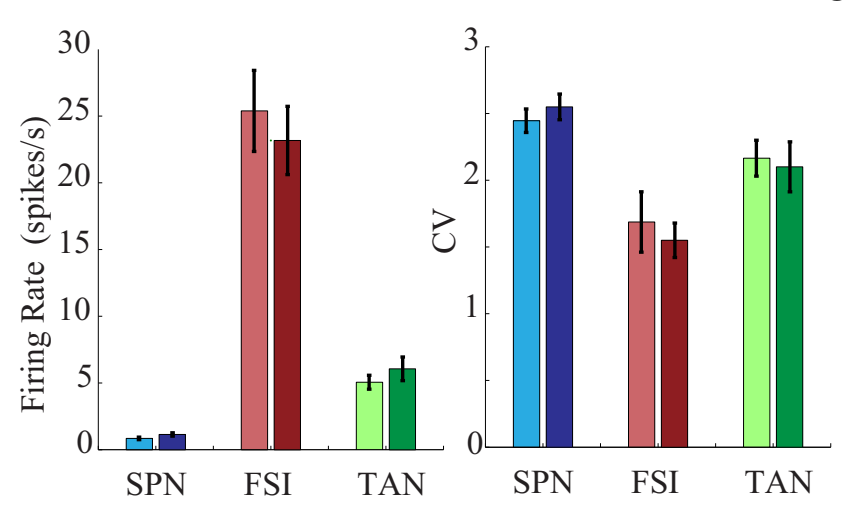

C

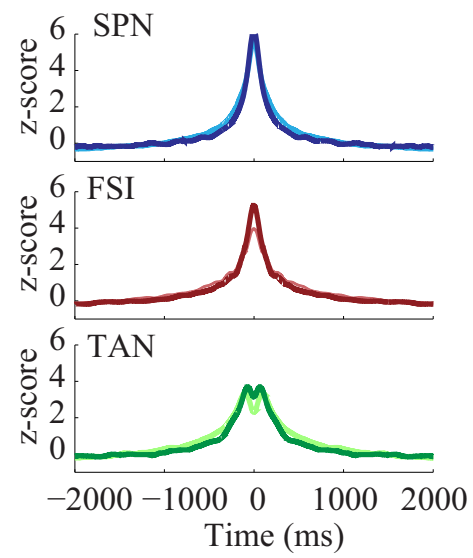

D
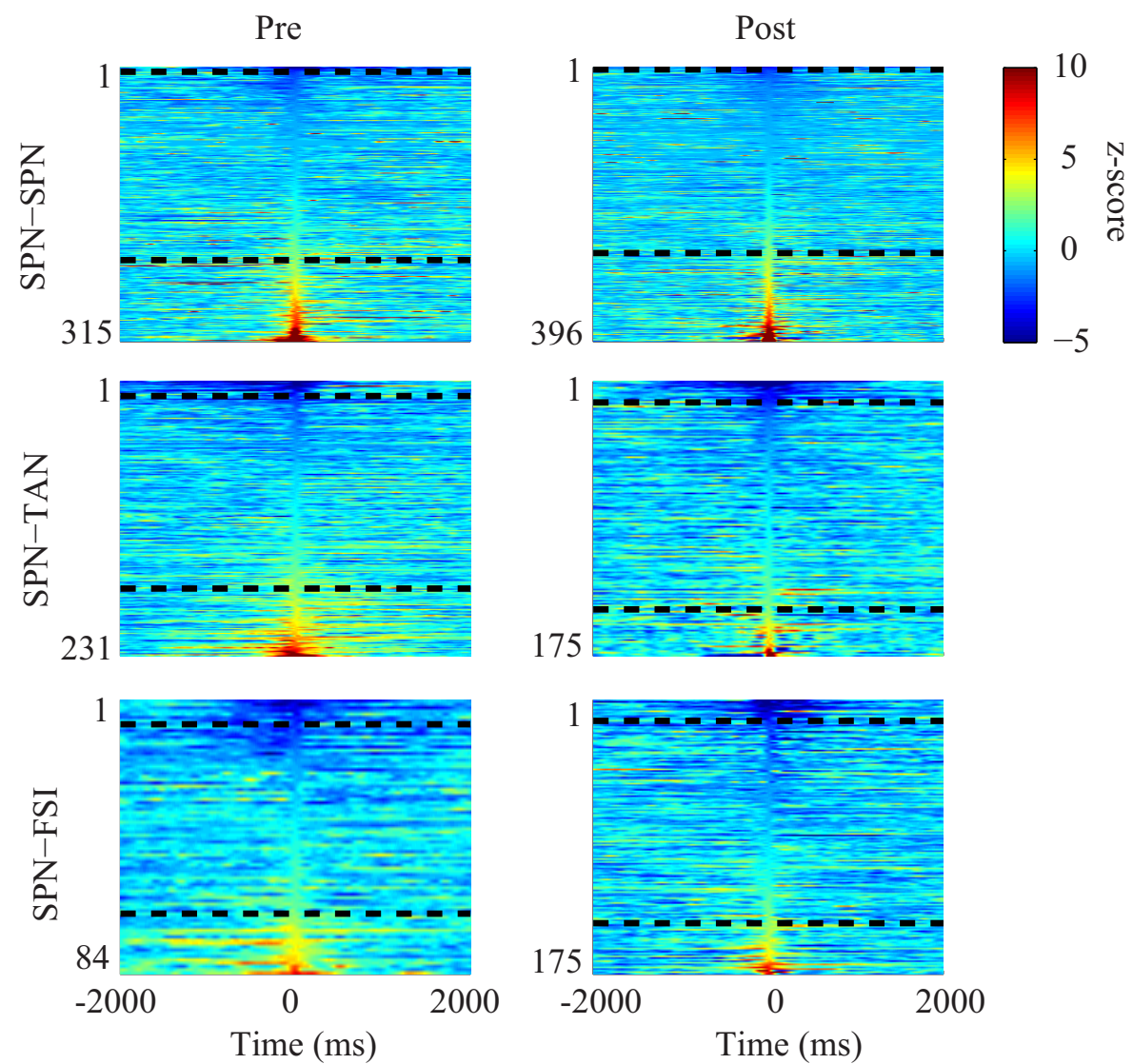

Figure 2. Bicuculline-induced changes in single neuron activity and interactions. $\boldsymbol{A}$, Mean firing rates, ( $\boldsymbol{B}$ ) CVs, and ( $\boldsymbol{C}$ ) autocorrelation functions of SPNs (blue), FSIs (red), and TANs (green), pre(light colors) and post- (dark colors) bicuculline injection (error bars, 1 SEM). D, Cross-correlation functions of all the simultaneously recorded single-unit pairs pre- (left) and post- (right) injection. Significance lines of positive (bottom) and negative (top) correlations are marked by dashed black lines.

group. We examined the change in the spiking activity in each PLTH given the relationship between neuronal activity and behavior (Fig. $7 A, B)$. A minority $(12 / 39,31 \%)$ of the neurons differed in their LFP spike locked activity between the two LFP groups, indicating a behavioral context dependency (Fig. $7 \mathrm{~A}, \mathrm{C}$ ). The largest group from out of these neurons was associated with movement termination (Fig. 7D). Most of the neurons displaying LFP spike-locked activity were independent of movement termination $(69 \%, 27 / 39$; Fig. 7 B, C). These neurons were associated with other components of movement, and only a small minority was related to movement termination (Fig. 7D), suggesting that individual neurons encode both normal movement and abnormal LFP spike-dependent changes independently of each other.

\section{Discussion}

In this study we explored the effect of NAc core disinhibition on the rat behavior and the underlying neuronal activity. We used a wireless acquisition system, which enabled the animals to move unrestrained within a large behavioral arena while continuously recording both electrophysiological and kinematic signals. This approach enabled precise synchronization of the different constituents of the neurophysiological signal and the corresponding 
A

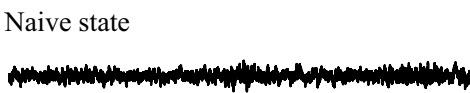

Hyperactivity

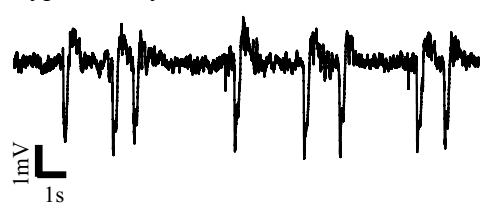

D

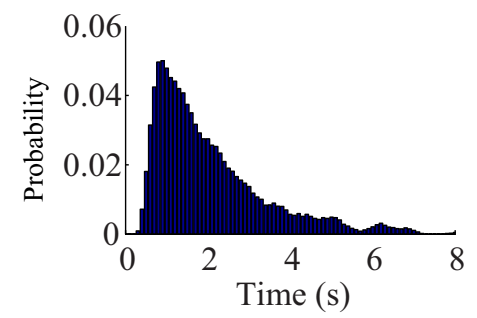

B

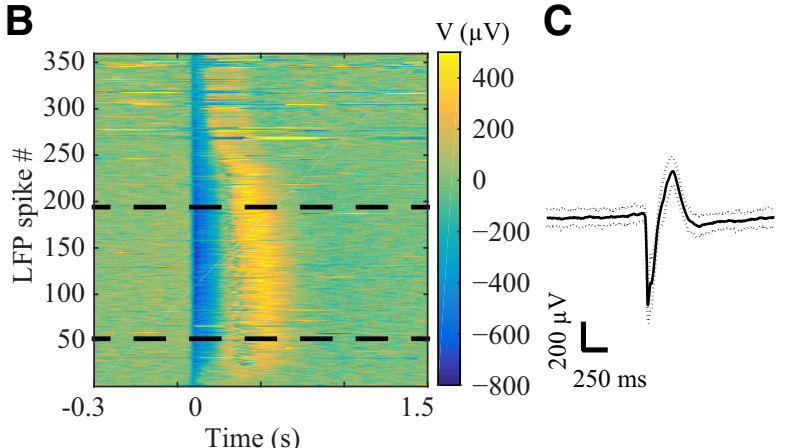

E

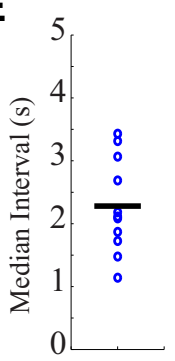

$\mathbf{F}$

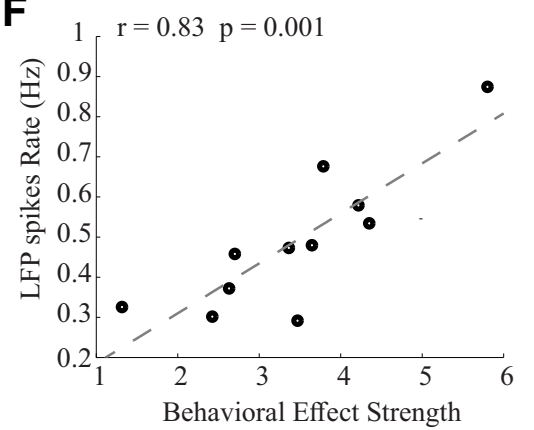

Figure 3. Bicuculline-induced changes in the LFP signal. $A$, Example of the LFP signal pre- (top) and post- (bottom) bicuculline injection. $\boldsymbol{B}$, Shapes of all the LFP spikes recorded over a single session. $\boldsymbol{C}$, Mean shape of the LFP spike during the stable period (solid black line) from the session shown in $\boldsymbol{B}$. $\boldsymbol{D}$, Inter-LFP-spike interval histogram taken from a single session. $\boldsymbol{E}$, Medians of the inter-LFP-spikes interval histograms (black line, mean of medians). $\boldsymbol{F}$, LFP spike rate as a function of behavioral effect strength (dashed line, linear regression).

behavior on multiple temporal scales. On the macro-scale, disinhibition resulted in hyperactivity without any abnormal movements: the animals moved within the behavioral arena without the transition to rest typical of naive animals. The magnitude of hyperactivity was animal-dependent, because the activity level during the hyperactive macro-state was dependent on the activity baseline level during the naive state of the same animal. The macro-scale behavioral state transition was not associated with major changes in either the tonic activity of individual neurons or their interactions. A major change, however, was observed on the neuronal population level: the LFP displayed large deflections (LFP spikes) whose frequency was linearly related to the magnitude of the behavioral effect. On the micro-scale, the rats' activity did not consist of continuous locomotion but rather included transitions between movement and non-movement microstates (episodes). The transitions between the microstates tended to be encoded by the neuronal population as individual LFP spikes accompanied by finely-tuned sparse activity modulations of single neurons, encoding different microstates related to movement while adhering to the same distribution as seen in the neuronal population of the naive animals.

Although the pathophysiology underlying ADHD is still largely unknown, multiple converging evidence from human patients studies point to the prominent role of the corticobasal ganglia pathway (Giedd et al., 2006). Anatomical evidence indicates a decrease in the volume of the striatum and in particular the ventral striatum (Carmona et al., 2009; Qiu et al., 2009; Greven et al., 2015). Physiological evidence (fMRI) suggests differences in activation (Durston et al., 2003) and responsiveness (Scheres et al., 2007; Plichta and Scheres, 2014) of these brain areas. In line with these results, early studies in animals showed that experimental manipulation of the NAc core changes their overall activity. Local application of GABA antagonists (Wachtel and Andén, 1978; Morgenstern et al., 1984), as well as glutamate agonists (Arnt, 1981) induces hyperactivity whereas glutamate antagonists (Mogenson and Nielsen, 1984; Swanson and Kalivas, 2000) and GABA agonists (Jones et al., 1981; Wong et al., 1991) induce hypoactivity.

Hyperactivity, a key symptom in many ADHD patients (American Psychiatric Association, 2013), was expressed in this study as enhanced normal behavior including locomotion, exploration, and other behaviors which were indistinguishable from the repertoire of the naive animals. This persistent hyperactivity expressed for a prolonged period following the disinhibition differed from the short-term novelty-associated hyperactivity of spontaneous hypertensive rats (Knardahl and Sagvolden, 1979). The normal movements expressed following disinhibition differed from the abnormal movements expressed by animal models after dopaminergic modulation, which are associated with dyskinesia and stereotypy (Fog, 1970; Costall and Naylor, 1976). The hyperkinetic symptoms subsequent to disinhibition were expressed solely as hyperactivity and there were neither abnormal individual movements nor abnormal movement sequences. The ongoing behavior during the macro-scale hyperactivity state was assembled from multiple micro-scale transitions between movements separated by brief non-movement periods. Frequent shifts between activities are common in many ADHD patients and are related to difficulties in sustaining attention. Similar attentiondeficit-like symptoms have been reported in primates using disinhibition in the globus pallidus externus (GPe; Grabli et al., 2004). Transitions between behavioral states also exist during these animals' normal behavior, as part of the normal repertoire (Markowitz et al., 2018). This supports the notion that behavioral transitions, which are attributed to the attentional state, may merely be another aspect of normal behavior, which is overexpressed during ADHD.

Disinhibition of different functional territories of the basal ganglia leads to a wide range of excessive movement (hyperki- 
A
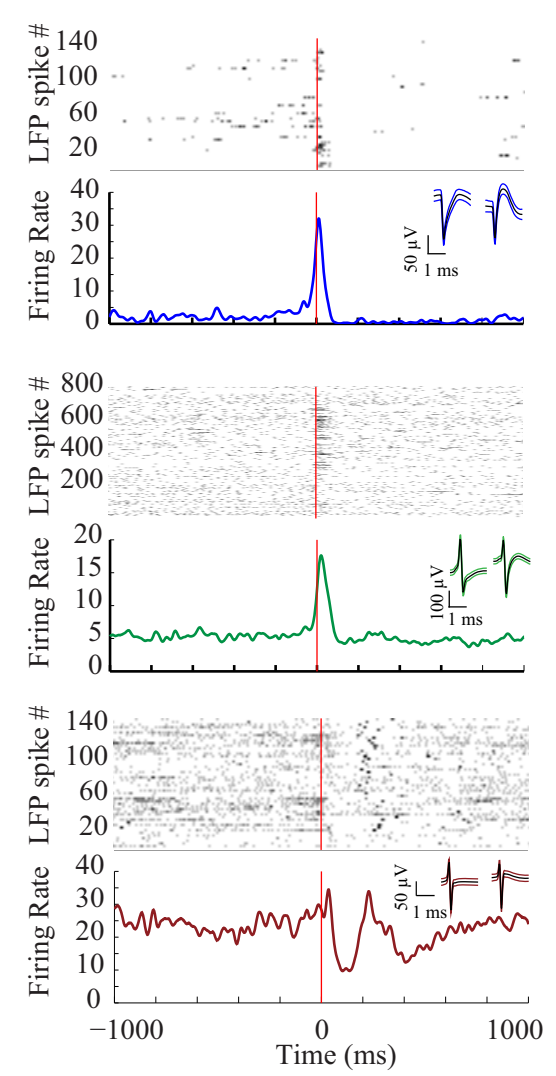

B
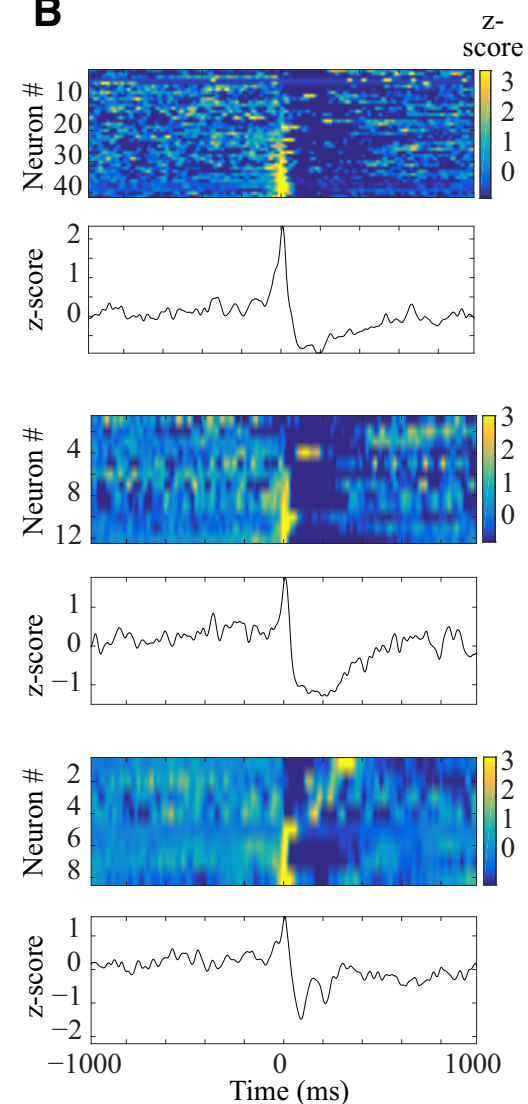

C

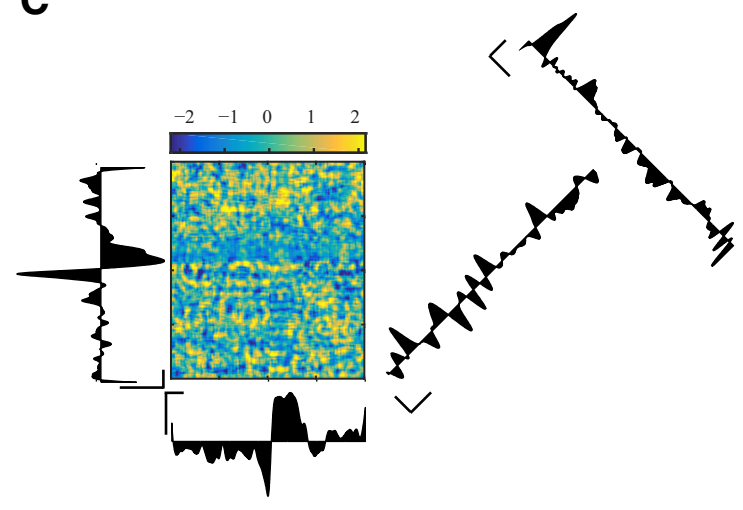

D

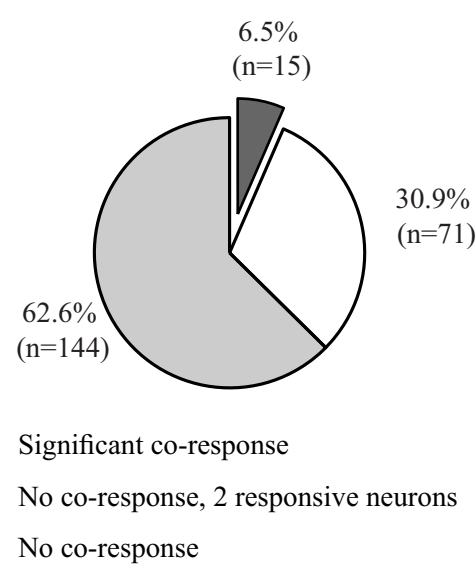

Figure 4. Single-unit activity around LFP spikes. A, Examples of peri-LFP-spike raster plots (top) and histograms (bottom) of individual SPN (top, blue), TAN (middle, green), and FSI (bottom, red) neurons. The inset within each histogram shows the mean spike shape using zero-phase (left) and nonlinear phase (right) filters. B, Matrices of single-unit peri-LFP-spike histograms (top) and their mean shape (bottom). C, Joint peri-LFP-spike histogram. Scale: Y, 2 SDs; X, $200 \mathrm{~ms}$. D, Co-response types based on the JPLTHs calculated for all pairs.

netic) and behavioral (hyperbehavioral) symptoms (Tarsy et al., 1978; Morgenstern et al., 1984; Grabli et al., 2004; Worbe et al., 2009). Hyperactivity in the form of increased locomotion has been reported in studies using disinhibition of limbic territories in rats, including the NAc (Wachtel and Andén, 1978; Jones et al., 1981; Morgenstern et al., 1984) and the ventral pallidum (Mogenson and Nielsen, 1983; Austin and Kalivas, 1990), and in primates (Worbe et al., 2009). In addition, disinhibition of the NAc shell led to emotional dysregulation (Stratford and Kelley, 1997; Lopes et al., 2012), which may contribute indirectly to the observed changes in locomotion. Multiple different hyperkinetic states have been evoked using similar manipulations in the sen- sorimotor basal ganglia: disinhibition of the sensorimotor striatum leads to motor tics (Tarsy et al., 1978; McCairn et al., 2009; Bronfeld et al., 2013; Pogorelov et al., 2015; Klaus and Plenz, 2016), downstream disinhibition in the sensorimotor GPe leads to chorea (Grabli et al., 2004; Bronfeld et al., 2010) and disinhibition of the striatum in cats (Yamada et al., 1995), and the motor thalamus in primates (Guehl et al., 2000) leads to dystonia. The disinhibition of sensorimotor territories share common properties: they lead to the expression of different hyperkinetic symptoms in the form of abnormal movements, which are not part of the normal animal's repertoire, are specific to body part, and are expressed unilaterally contralateral to the injected hemisphere. 


\section{A}
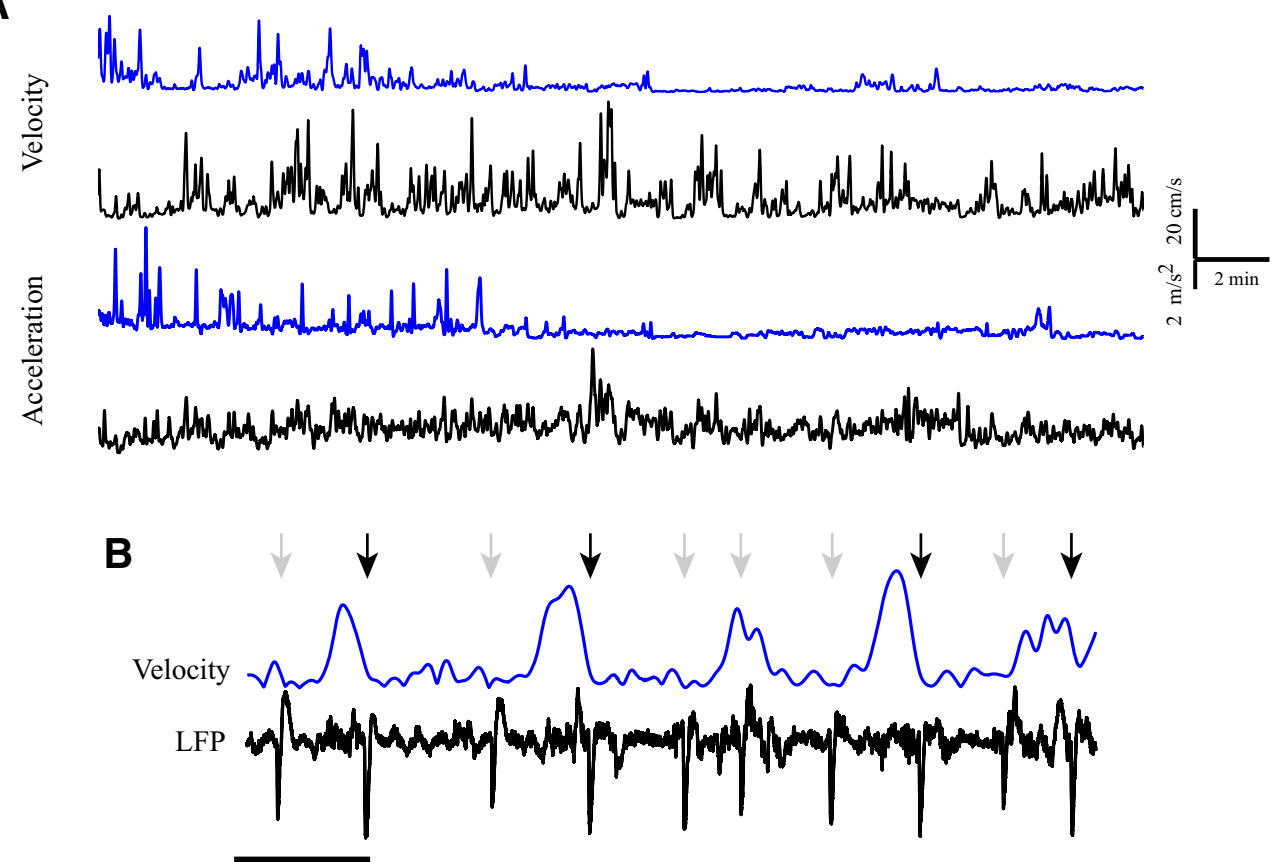

$5 \mathrm{~s}$

C

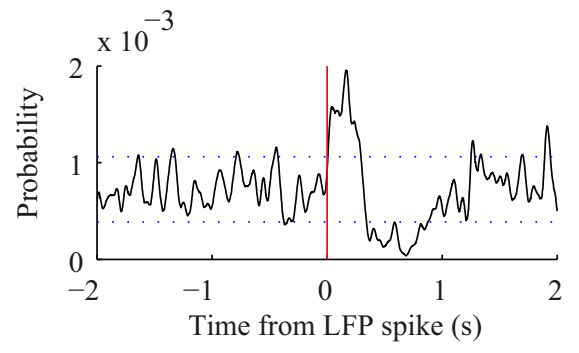

D
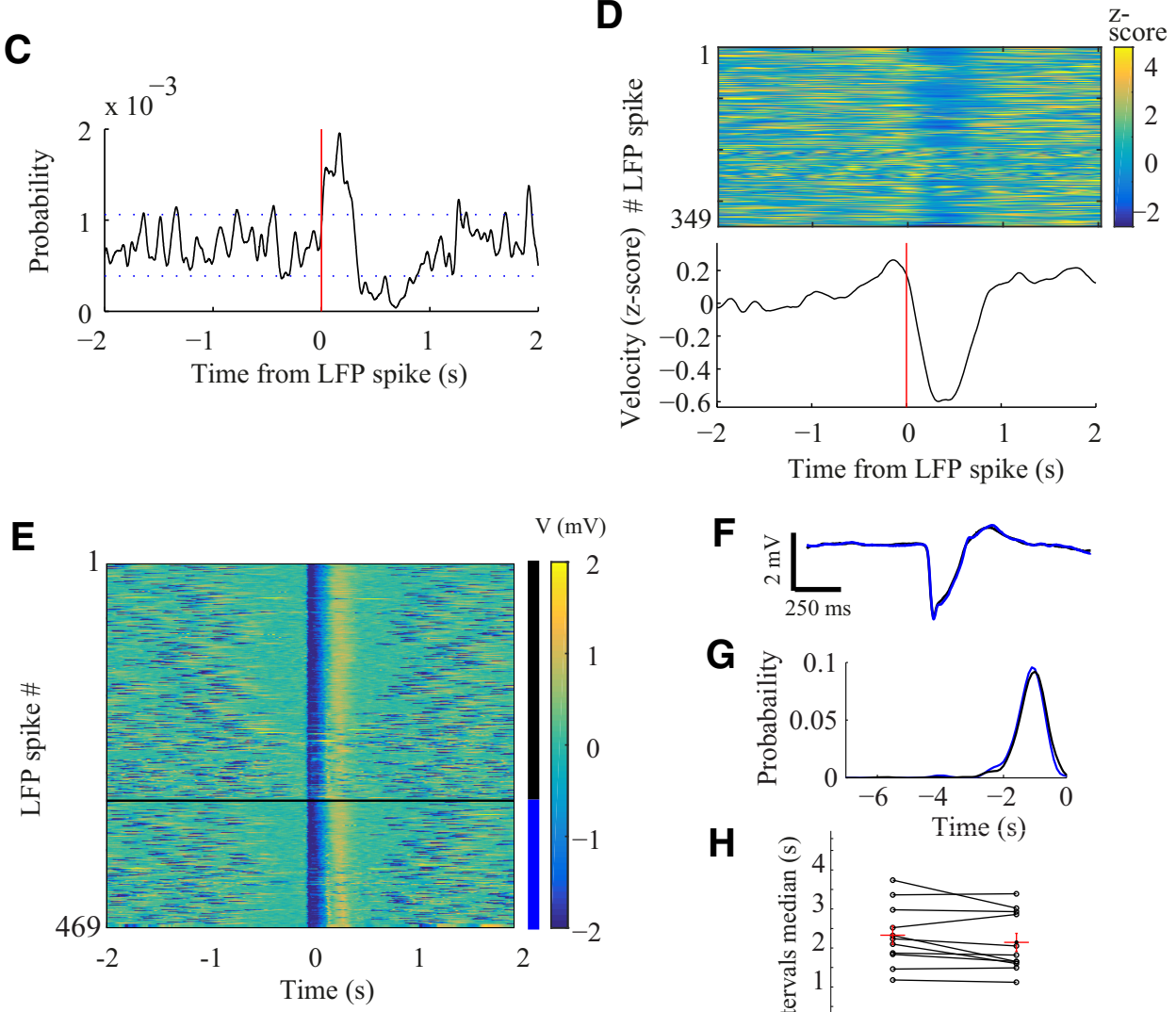

F

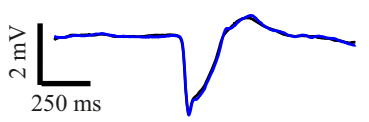

G

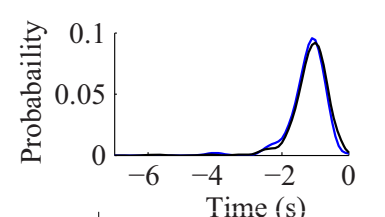

H

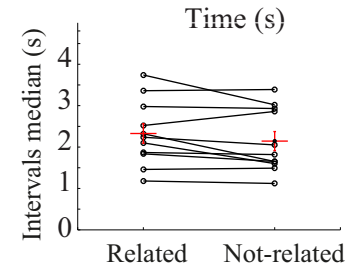

Figure 5. Micro-scale behavior and neuronal activity. $\boldsymbol{A}$, Example of the video-based velocity and sensor-based acceleration pre- (blue) and post- (black) injection. $\boldsymbol{B}$, Example of the animal's velocity (blue, top) and the corresponding LFP signal (black, bottom). Arrows mark the LFP time related (black arrow) and unrelated (gray arrow) to movement termination. C, Peri LFP-spike movement-termination histogram. D, LFP spike-triggered velocity (top) and the mean LFP triggered velocity (bottom). E, LFP spike shapes separated into movement-termination related (blue, bottom) and unrelated (black, top) and their $(\boldsymbol{F})$ mean shape (related, blue; unrelated, black). $\mathbf{G}$, Histograms of the intervals between movement-termination related (blue) and unrelated (black) LFP spikes and their $(\boldsymbol{H})$ medians calculated for 11 sessions (red, mean of the sessions). 


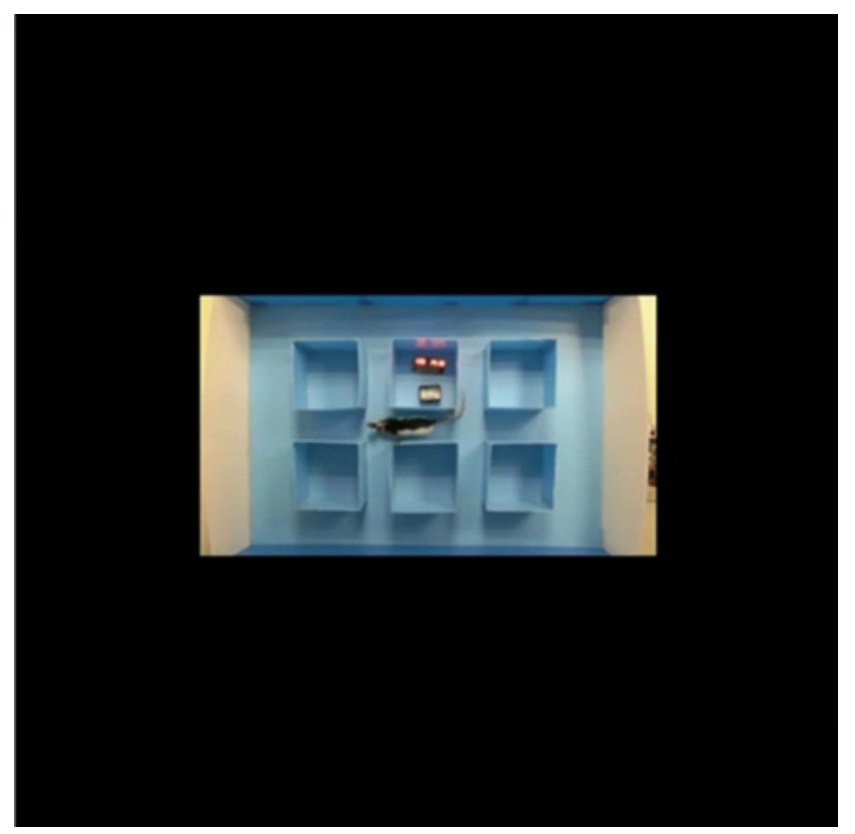

Movie 2. Behavior microstructure and its relationship to the LFP. The time series representing the LFP spike times is presented as a series of auditory beeps, which are overlaid on the video stream.
Similar manipulations in the limbic part of the striatum lead to hyperactivity expressed as increased normal behavior, which is nonspecific to a body part and is expressed bilaterally. Disinhibition of the sensorimotor territory in the striatum was shown to lead to the appearance of LFP spikes and phase-locked individual neuronal activity which were correlated with the appearance of individual tics (Bronfeld et al., 2013; Israelashvili and Bar-Gad, 2015; Pogorelov et al., 2015; Klaus and Plenz, 2016). The timing of individual LFP spikes was correlated with unitary behavioral events, individual tics, resembling the correlation to individual movement terminations in the current study. Our results support the hypothesis that striatal disinhibition leads to the release of unwanted motor plans (Albin and Mink, 2006). The disinhibition hypothesis, which in the sensorimotor striatum results in the expression of motor tics, may be extended to the NAc in which similar loss of inhibition leads to behavioral switches and the increased performance of motor plans. The source of this abnormal disinhibition, i.e., SPN collaterals (Chuhma et al., 2011), FSI feedforward inhibition (Koos et al., 2004), or GPe feedback inhibition (Mallet et al., 2012), however, cannot be distinguished by this study. The similarity of network manipulation across the striatal territories and the resulting underlying neuronal activity fits well with the high comorbidity $(\sim 50 \%)$ of tic expression and hyperactivity in Tourette syndrome patients (Schlaggar and Mink, 2003), hinting at similar fundamental neuronal changes underlying the two sets of symptoms. These different symptoms also support the functional distinction between territories in which the sensorimotor territory is associated with the form of individual movement primitives (Wiltschko et al., 2015; Klaus et al., 2017; Markowitz et al., 2018), whereas the limbic territory is

A

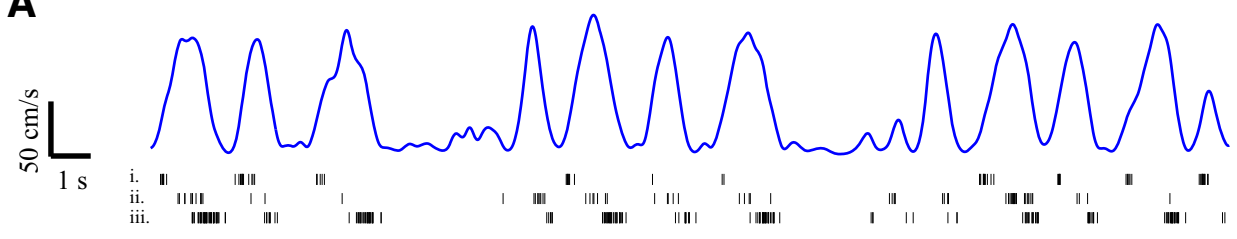

B

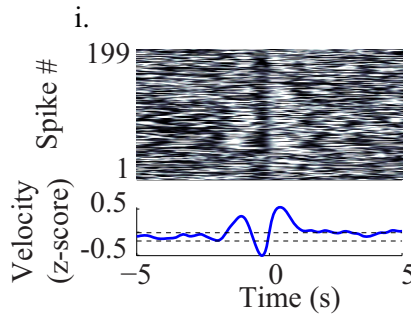

C

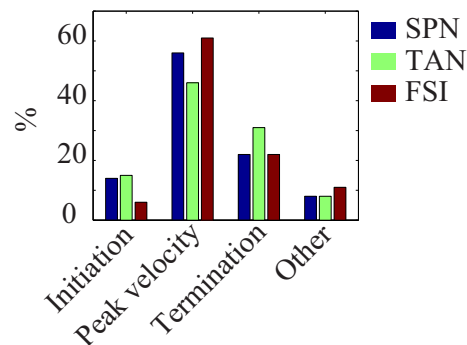

ii.

673
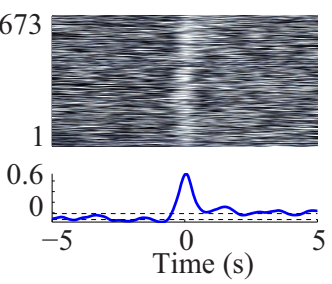

iii.

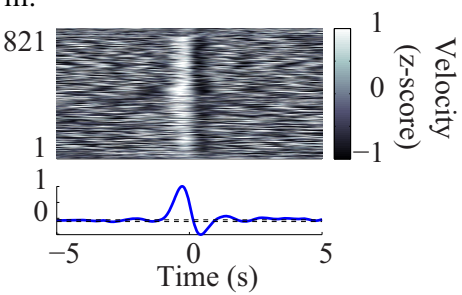

D Pre injection Post injection

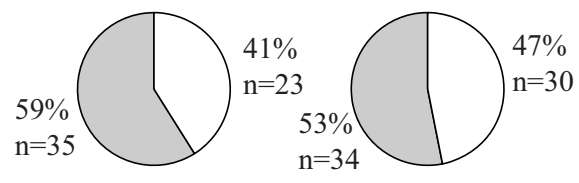

$\square$ Movement related neurons

$\square$ Movement unrelated neurons

Figure 6. Single-unit activity relation to micro-scale behavior. $\boldsymbol{A}$, Spike trains of three ventromedial striatum neurons plotted below the locomotion velocity. $\boldsymbol{B}$, Velocity aligned to the spike times (top) and the mean spike-triggered velocity (bottom) of the spike trains of the session shown in $\boldsymbol{A}$ showing that each neuron encodes a different component of movement (i, movement initiation; ii, peak velocity; iii, movement termination). C, Fraction of neurons associated with different components of movement, grouped by cell type (blue, SPN; green, TAN; red, FSI). D, Fraction of movement related and unrelated neurons pre- (left) and post- (right) injection. 
A PLTH

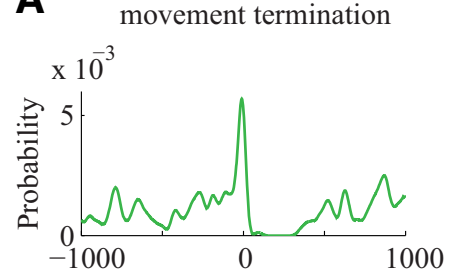

B

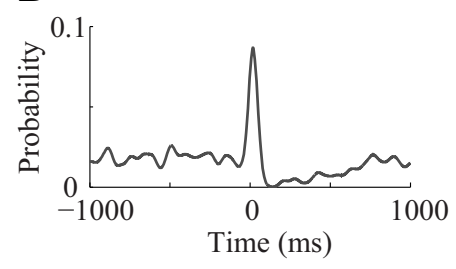

C

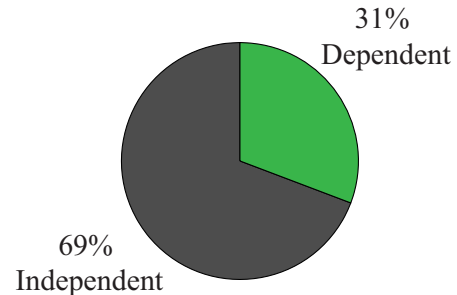

PLTH

non movement termination
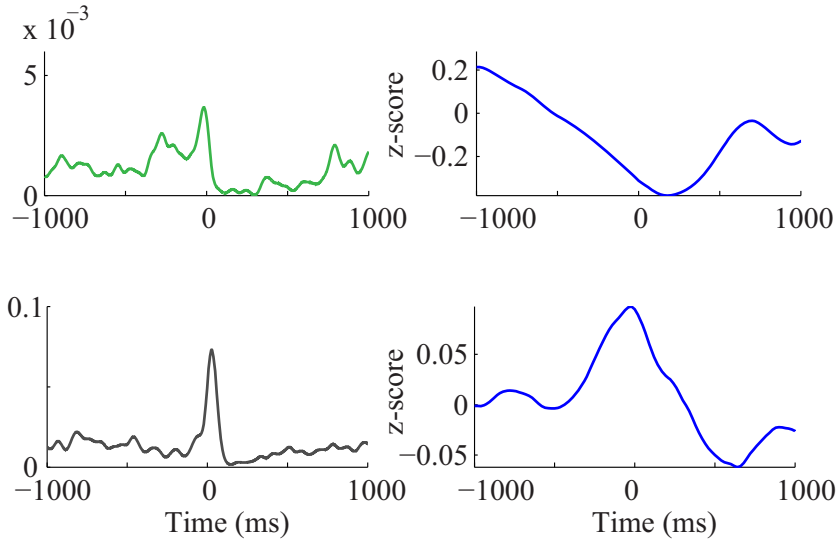

D

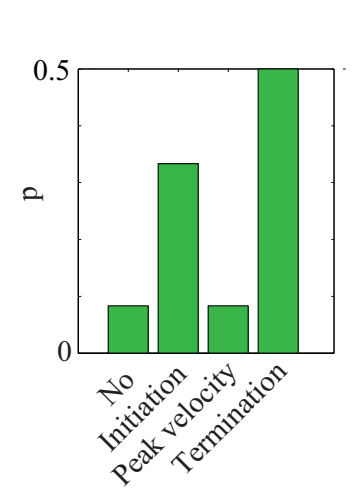

Spike Triggered Velocity 000

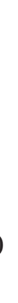

\section{D}

Figure 7. Three-way interaction between single-unit activity, LFP spikes and micro-scale behavior. $\boldsymbol{A}, \boldsymbol{B}$, Examples of behavioral context-dependent ( $\boldsymbol{A}$ ) and independent ( $\boldsymbol{B}$ ) LFP-spike locked activity of two single units illustrated by the peri-movement termination related (left) and unrelated (middle) LFP-spike histograms and the corresponding spike-triggered velocity (right). $\boldsymbol{C}$, Fraction of behavioral context dependent and independent LFP-spike locked activity. $\boldsymbol{D}$, Fraction of the neurons related to different components of movement in each group (green, behavioral context-dependent; gray, behavioral context independent LFP-spike locked activity).

associated with the number of movement primitives and switches between them.

The mechanism by which GABAergic disinhibition leads to macro- and micro-scale changes in behavior may be attributed to changes in the properties of either (1) the intrastriatal network, or (2) the extra-striatal inputs or their processing by the striatal neurons. The lack of changes in macro-scale activity or the interaction of the striatal neurons, together with similar neuronal encoding on the micro-scale do not support the likelihood of tonic alteration of the intrastriatal network as the basis for macro-scale state change. Studies manipulating the tonic inputs to the NAc have reported similar behavioral results using both glutamatergic (Arnt, 1981) and dopaminergic (Jones et al., 1981) agents. These results lend weight to the notion of a mechanism based on alteration of the input to the striatum or on the responsiveness to such input. This mechanism was addressed by an in vitro study investigating the NAc synaptic transmission using extracellular field recordings (Buckby and Lacey, 2001), which demonstrated that cortical input forms the principal drive of regenerative field changes, resembling the observed LFP spikes in our study following administration of $\mathrm{GABA}_{\mathrm{A}}$ antagonists. A recent study demonstrated, in vivo, that the timing of unitary behavioral events associated with LFP spikes (motor tics) was determined by the cortical input to the striatal network (Israelashvili and Bar-Gad, 2015), supporting the idea of an extra-striatal input-based mechanism. However, further studies are required to fully determine the source of the LFP spikes and their relation to the observed symptoms.
Disinhibition of the NAc core leads to neuronal changes consistent with those observed after disinhibition of the dorsal striatum but with different behavioral symptoms; i.e., macro-scale hyperactivity and micro-scale activity switches. These results emphasize the unique role of the NAc in controlling the overall level of activity and the switches between movement primitives, a function that is synergistic with the control of the individual movement primitives by the dorsolateral striatum. These complementary roles in normal behavior may also underlie the expression of the very different, yet highly comorbid symptoms of Tourette syndrome and ADHD.

\section{References}

Aertsen AM, Gerstein GL, Habib MK, Palm G (1989) Dynamics of neuronal firing correlation: modulation of "effective connectivity". J Neurophysiol 61:900-917.

Albin RL, Mink JW (2006) Recent advances in Tourette syndrome research. Trends Neurosci 29:175-182.

Al-Hasani R, McCall JG, Shin G, Gomez AM, Schmitz GP, Bernardi JM, Pyo CO, Park SI, Marcinkiewcz CM, Crowley NA, Krashes MJ, Lowell BB, Kash TL, Rogers JA, Bruchas MR (2015) Distinct subpopulations of nucleus accumbens dynorphin neurons drive aversion and reward. Neuron 87:1063-1077.

American Psychiatric Association (2013) Diagnostic and statistical manual of mental disorders: DSM-5. Washington, DC; London: American Psychiatric.

Arnt J (1981) Hyperactivity following injection of a glutamate agonist and 6, 7-ADTN into rat nucleus accumbens and its inhibition by THIP. Life Sci 28:1597-1603.

Austin MC, Kalivas PW (1990) Enkephalinergic and GABAergic modula- 
tion of motor activity in the ventral pallidum. J Pharmacol Exp Ther 252:1370-1377.

Bar-Gad I, Ritov Y, Vaadia E, Bergman H (2001) Failure in identification of overlapping spikes from multiple neuron activity causes artificial correlations. J Neurosci Methods 107:1-13.

Bronfeld M, Belelovsky K, Erez Y, Bugaysen J, Korngreen A, Bar-Gad I (2010) Bicuculline-induced chorea manifests in focal rather than globalized abnormalities in the activation of the external and internal globus pallidus. J Neurophysiol 104:3261-3275.

Bronfeld M, Belelovsky K, Bar-Gad I (2011) Spatial and temporal properties of tic-related neuronal activity in the cortico-basal ganglia loop. J Neurosci 31:8713-8721.

Bronfeld M, Yael D, Belelovsky K, Bar-Gad I (2013) Motor tics evoked by striatal disinhibition in the rat. Front Syst Neurosci 7:50.

Buckby LE, Lacey MG (2001) Epileptiform activity in the nucleus accumbens induced by GABAA receptor antagonists in rat forebrain slices is of cortical origin. Exp Brain Res 141:146-152.

Carmona S, Proal E, Hoekzema EA, Gispert JD, Picado M, Moreno I, Soliva JC, Bielsa A, Rovira M, Hilferty J, Bulbena A, Casas M, Tobeña A, Vilarroya $\mathrm{O}$ (2009) Ventro-striatal reductions underpin symptoms of hyperactivity and impulsivity in attention-deficit/hyperactivity disorder. Biol Psychiatry 66:972-977.

Chuhma N, Tanaka KF, Hen R, Rayport S (2011) Functional connectome of the striatal medium spiny neuron. J Neurosci 31:1183-1192.

Costall B, Naylor RJ (1975) The behavioural effects of dopamine applied intracerebrally to areas of the mesolimbic system. Eur J Pharmacol 32:8792.

Costall B, Naylor RJ (1976) Dissociation of stereotyped biting responses and oro-bucco-lingual dyskinesias. Eur J Pharmacol 36:423-429.

Costall B, Domeney AM, Naylor RJ (1984) Locomotor hyperactivity caused by dopamine infusion into the nucleus accumbens of rat brain: specificity of action. Psychopharmacology 82:174-180.

Cui G, Jun SB, Jin X, Pham MD, Vogel SS, Lovinger DM, Costa RM (2013) Concurrent activation of striatal direct and indirect pathways during action initiation. Nature 494:238-242.

Dalia A, Uretsky NJ, Wallace LJ (1998) Dopaminergic agonists administered into the nucleus accumbens: effects on extracellular glutamate and on locomotor activity. Brain Res 788:111-117.

Durston S, Tottenham NT, Thomas KM, Davidson MC, Eigsti IM, Yang Y, Ulug AM, Casey BJ (2003) Differential patterns of striatal activation in young children with and without ADHD. Biol Psychiatry 53:871-878.

Floresco SB, Montes DR, Tse MMT, van Holstein M (2018) Differential contributions of nucleus accumbens subregions to cue-guided risk/reward decision making and implementation of conditional rules. J Neurosci 38:1901-1914.

Fog R (1970) Behavioural effects in rats of morphine and amphetamine and of a combination of the two drugs. Psychopharmacologia 16:305-312.

Giedd JN, Blumenthal J, Molloy E, Castellanos FX (2006) Brain imaging of attention deficit/hyperactivity disorder. Ann N Y Acad Sci 931:33-49.

Grabli D, McCairn K, Hirsch EC, Agid Y, Féger J, François C, Tremblay L (2004) Behavioural disorders induced by external globus pallidus dysfunction in primates: I. behavioural study. Brain 127:2039-2054.

Greven CU, Bralten J, Mennes M, O’Dwyer L, Van Hulzen KJ, Rommelse N, Schweren LJ, Hoekstra PJ, Hartman CA, Heslenfeld D, Oosterlaan J, Faraone SV, Franke B, Zwiers MP, Arias-Vasquez A, Buitelaar JK (2015) Developmentally stable whole-brain volume reductions and developmentally sensitive caudate and putamen volume alterations in those with attention-deficit/hyperactivity disorder and their unaffected siblings. JAMA Psychiatry 72:490-499.

Guehl D, Burbaud P, Boraud T, Bioulac B (2000) Bicuculline injections into the rostral and caudal motor thalamus of the monkey induce different types of dystonia. Eur J Neurosci 12:1033-1037.

Heilbronner SR, Rodriguez-Romaguera J, Quirk GJ, Groenewegen HJ, Haber SN (2016) Circuit-based corticostriatal homologies between rat and primate. Biol Psychiatry 80:509-521.

Heimer L, Zahm DS, Churchill L, Kalivas PW, Wohltmann C (1991) Specificity in the projection patterns of accumbal core and shell in the rat. Neuroscience 41:89-125.

Israelashvili M, Bar-Gad I (2015) Corticostriatal divergent function in determining the temporal and spatial properties of motor tics. J Neurosci 35:16340-16351.

Jones DL, Mogenson GJ, Wu M (1981) Injections of dopaminergic, cholin- ergic, serotoninergic and GABAergic drugs into the nucleus accumbens: effects on locomotor activity in the rat. Neuropharmacology 20:29-37.

Klaus A, Plenz D (2016) A low-correlation resting state of the striatum during cortical avalanches and its role in movement suppression. PLoS Biol 14:e1002582.

Klaus A, Martins GJ, Paixao VB, Zhou P, Paninski L, Costa RM (2017) The spatiotemporal organization of the striatum encodes action space. Neuron 95:1171-1180.e7.

Knardahl S, Sagvolden T (1979) Open-field behavior of spontaneously hypertensive rats. Behav Neural Biol 27:187-200.

Koos T, Tepper JM, Wilson CJ (2004) Comparison of IPSCs evoked by spiny and fast-spiking neurons in the neostriatum. J Neurosci 24:7916-7922.

Lopes AP, Ganzer L, Borges AC, Kochenborger L, Januário AC, Faria MS, Marino-Neto J, Paschoalini MA (2012) Effects of GABA ligands injected into the nucleus accumbens shell on fear/anxiety-like and feeding behaviours in food-deprived rats. Pharmacol Biochem Behav 101:41-48.

Maldonado-Irizarry CS, Kelley AE (1995) Excitotoxic lesions of the core and shell subregions of the nucleus accumbens differentially disrupt body weight regulation and motor activity in rat. Brain Res Bull 38:551-559.

Maldonado-Irizarry CS, Kelley AE (1994) Differential behavioral effects following microinjection of an NMDA antagonist into nucleus accumbens subregions. Psychopharmacology 116:65-72.

Mallet N, Micklem BR, Henny P, Brown MT, Williams C, Bolam JP, Nakamura KC, Magill PJ (2012) Dichotomous organization of the external globus pallidus. Neuron 74:1075-1086.

Markowitz JE, Gillis WF, Beron CC, Neufeld SQ, Robertson K, Bhagat ND, Peterson RE, Peterson E, Hyun M, Linderman SW, Sabatini BL, Datta SR (2018) The striatum organizes 3D behavior via moment-to-moment action selection. Cell 174:44-58.e17.

McCairn KW, Bronfeld M, Belelovsky K, Bar-Gad I (2009) The neurophysiological correlates of motor tics following focal striatal disinhibition. Brain 132:2125-2138.

McGeorge AJ, Faull RL (1989) The organization of the projection from the cerebral cortex to the striatum in the rat. Neuroscience 29:503-537.

Mogenson GJ, Nielsen MA (1983) Evidence that an accumbens to subpallidal GABAergic projection contributes to locomotor activity. Brain Res Bull 11:309-314.

Mogenson GJ, Nielsen M (1984) Neuropharmacological evidence to suggest that the nucleus accumbens and subpallidal region contribute to exploratory locomotion. Behav Neural Biol 42:52-60.

Mogenson GJ, Jones DL, Yim CY (1980) From motivation to action: functional interface between the limbic system and the motor system. Prog Neurobiol 14:69-97.

Morgenstern R, Mende T, Gold R, Lemme P, Oelssner W (1984) Druginduced modulation of locomotor hyperactivity induced by picrotoxin in nucleus accumbens. Pharmacol Biochem Behav 21:501-506.

Oran Y, Bar-Gad I (2018) Loss of balance between striatal feedforward inhibition and corticostriatal excitation leads to tremor. J Neurosci 38: $1699-1710$

Otsu N (1979) A threshold selection method from gray-level histograms. IEEE Trans Syst Man Cybern 9:62-66.

Plichta MM, Scheres A (2014) Ventral-striatal responsiveness during reward anticipation in ADHD and its relation to trait impulsivity in the healthy population: a meta-analytic review of the fMRI literature. Neurosci Biobehav Rev 38:125-134.

Pogorelov V, Xu M, Smith HR, Buchanan GF, Pittenger C (2015) Corticostriatal interactions in the generation of tic-like behaviors after local striatal disinhibition. Exp Neurol 265:122-128.

Pycock CJ, Horton RW (1979) Dopamine-dependent hyperactivity in the rat following manipulation of GABA mechanisms in the region of the nucleus accumbens. J Neural Transm 45:17-33.

Qiu A, Crocetti D, Adler M, Mahone EM, Denckla MB, Miller MI, Mostofsky SH (2009) Basal ganglia volume and shape in children with attention deficit hyperactivity disorder. Am J Psychiatry 166:74-82.

Scheres A, Milham MP, Knutson B, Castellanos FX (2007) Ventral striatal hyporesponsiveness during reward anticipation in attention-deficit/hyperactivity disorder. Biol Psychiatry 61:720-724

Schlaggar BL, Mink JW (2003) Movement disorders in children. Pediatr Rev 24:39-51.

Schultz W, Romo R (1988) Neuronal activity in the monkey striatum during the initiation of movements. Exp Brain Res 71:431-436. 
Sowell ER, Kan E, Yoshii J, Thompson PM, Bansal R, Xu D, Toga AW, Peterson BS (2008) Thinning of sensorimotor cortices in children with Tourette syndrome. Nat Neurosci 11:637-639.

Stratford TR, Kelley AE (1997) GABA in the nucleus accumbens shell participates in the central regulation of feeding behavior. J Neurosci 17:4434-4440.

Swanson CJ, Kalivas PW (2000) Regulation of locomotor activity by metabotropic glutamate receptors in the nucleus accumbens and ventral tegmental area. J Pharmacol Exp Ther 292:406-414.

Tarsy D, Pycock CJ, Meldrum BS, Marsden CD (1978) Focal contralateral myoclonus produced by inhibition of GABA action in the caudate nucleus of rats. Brain 101:143-162.

Tremblay L, Worbe Y, Thobois S, Sgambato-Faure V, Féger J (2015) Selective dysfunction of basal ganglia subterritories: from movement to behavioral disorders. Mov Disord 30:1155-1170.

Volkow ND, Morales M (2015) The brain on drugs: from reward to addiction. Cell 162:712-725.

Voorn P, Gerfen CR, Groenewegen HJ (1989) Compartmental organization of the ventral striatum of the rat: immunohistochemical distribution of enkephalin, substance $\mathrm{P}$, dopamine, and calcium-binding protein. J Comp Neurol 289:189-201.

Wachtel H, Andén NE (1978) Motor activity of rats following intracerebral injections of drugs influencing GABA mechanisms. Naunyn Schmiedebergs Arch Pharmacol 302:133-139.

West EA, Carelli RM (2016) Nucleus accumbens core and shell differentially encode reward-associated cues after reinforcer devaluation. J Neurosci 36:1128-1139.

Wiltschko AB, Johnson MJ, Iurilli G, Peterson RE, Katon JM, Pashkovski SL, Abraira VE, Adams RP, Datta SR (2015) Mapping sub-second structure in mouse behavior. Neuron 88:1121-1135.

Wong LS, Eshel G, Dreher J, Ong J, Jackson DM (1991) Role of dopamine and GABA in the control of motor activity elicited from the rat nucleus accumbens. Pharmacol Biochem Behav 38:829-835.

Worbe Y, Baup N, Grabli D, Chaigneau M, Mounayar S, McCairn K, Féger J, Tremblay L (2009) Behavioral and movement disorders induced by local inhibitory dysfunction in primate striatum. Cereb Cortex 19: 1844-1856.

Yael D, Bar-Gad I (2017) Filter based phase distortions in extracellular spikes. PLoS One 12:e0174790.

Yael D, Zeef DH, Sand D, Moran A, Katz DB, Cohen D, Temel Y, Bar-Gad I (2013) Haloperidol-induced changes in neuronal activity in the striatum of the freely moving rat. Front Syst Neurosci 7:110.

Yael D, Vecht JJ, Bar-Gad I (2018) Filter based phase shifts distort neuronal timing information. eNeuro 5:ENEURO.0261-17.2018.

Yamada H, Fujimoto K, Yoshida M (1995) Neuronal mechanism underlying dystonia induced by bicuculline injection into the putamen of the cat. Brain Res 677:333-336.

Yoshida M, Nagatsuka Y, Muramatsu S, Niijima K (1991) Differential roles of the caudate nucleus and putamen in motor behavior of the cat as investigated by local injection of GABA antagonists. Neurosci Res 10:34-51. 